Testing the race inequality A simple correction procedure for fast guesses

Gondan, Matthias; Heckel, A.

Published in:

Journal of Mathematical Psychology

DOI:

10.1016/j.jmp.2008.08.002

Publication date:

2008

Document version

Publisher's PDF, also known as Version of record

Citation for published version (APA):

Gondan, M., \& Heckel, A. (2008). Testing the race inequality: A simple correction procedure for fast guesses. Journal of Mathematical Psychology, 52(5), 322-325. https://doi.org/10.1016/j.jmp.2008.08.002 
Theoretical note

\title{
Testing the race inequality: A simple correction procedure for fast guesses
}

\author{
Matthias Gondan*, Andreas Heckel \\ Department of Experimental Psychology, University of Regensburg, D-93050 Regensburg, Germany
}

\section{A R T I C L E I N F O}

\section{Article history:}

Received 21 February 2008

Received in revised form

5 August 2008

Available online 21 September 2008

\section{Keywords:}

Divided attention

Multisensory processing

Race model

\begin{abstract}
A B S T R A C T
In speeded response tasks with redundant signals, parallel processing of the redundant signals is generally tested using the so-called race inequality. The race inequality states that the distribution of fast responses for a redundant stimulus never exceeds the summed distributions of fast responses for the single stimuli. It has been pointed out that fast guesses (e.g. anticipatory responses) interfere with this test, and a correction procedure ('kill-the-twin' procedure) has been suggested. In this note we formally derive this procedure and extend it to the case in which redundant stimuli are presented with onset asynchrony. We demonstrate how the kill-the-twin procedure is used in a statistical test of the race model prediction.
\end{abstract}

(C) 2008 Elsevier Inc. All rights reserved.

\section{Introduction}

In a typical redundant target experiment, the same response is required for two classes of stimuli (e.g. auditory and visual stimuli, $A$ and $V$ ). When both stimuli are presented simultaneously (redundant targets, $A V$ ), participants usually respond faster than to the single stimuli. This effect has been termed 'redundant signals effect' or 'redundant target effect'. Two classes of models have been suggested to explain this effect, race models and coactivation models. Race models assume that the two stimuli are processed in separate channels. On redundant target trials, the faster channel elicits the response:

$T_{A V}={ }_{s t} \min \left(T_{A}, T_{V}\right)$.

We adopt the notation of Maris and Maris (2003), using $=_{s t}$ (stochastically equal) to denote that the distribution of $T_{A V}$ is the same as for the minimum of $T_{A}$ and $T_{V}$. It follows that a redundant target response, $A V$, occurs within a given $t$ if either the auditory or the visual subprocess has finished before $t$ :

$\left\{T_{A V} \leq t\right\}=\left\{T_{A} \leq t\right\} \cup\left\{T_{V} \leq t\right\}$.

Under the assumption that the processing time distributions for $T_{A}$ and $T_{V}$ can be estimated from the unimodal response time distributions ('context independence', Luce, 1986, p. 130), an upper limit for the response time distribution for $A V$ can be derived (Miller, 1982):

$P\left\{T_{A V} \leq t\right\} \leq P\left\{T_{A} \leq t\right\}+P\left\{T_{V} \leq t\right\}$.

\footnotetext{
* Corresponding author

E-mail address: matthias.gondan@psychologie.uni-regensburg.de (M. Gondan).
}

Inequality (3) can easily be extended to redundant stimuli presented with onset asynchrony. For example, if the visual stimulus is presented $\tau$ ms after the auditory stimulus, processing of $V$ starts with a delay of $\tau \mathrm{ms}: T_{A V(\tau)}={ }_{s t} \min \left(T_{A}, T_{V}+\tau\right)$. It follows (Miller, 1986) that:

$P\left\{T_{A V(\tau)} \leq t\right\} \leq P\left\{T_{A} \leq t\right\}+P\left\{T_{V}+\tau \leq t\right\}$.

If the visual stimulus is presented first, the subscripts $A$ and $V$ have to be exchanged. Inequalities (3) and (4) are somehow asymmetric, there is only one term on the left side, whereas there are two summands on the right side. Eriksen (1988) noted that this asymmetry raises problems if participants guess the onset of a target stimulus. Such guesses increase the observed amount of fast responses on both sides of the inequality. Since this increase is stronger on the right side with two summands than on the left side with only one term, a race model might erroneously be adopted; this has been demonstrated by Eriksen (1988) and Miller and Lopes (1991, Eq. 6). Eriksen suggested applying a socalled 'kill-the-twin' procedure in which the distribution of fast guesses is estimated from erroneous responses (e.g. 'catch' trials, $O$, in which no stimulus is presented); for each of these erroneous responses, a correct response of similar speed is eliminated before testing Inequality (3). This procedure is derived from a two-state model (Ollman, 1966; Yellott, 1971, "simple fast guess model") in which the participant guesses the onset of the next stimulus with probability $g$. With probability $1-g$, the participant responds to the stimulus. The observed response time distribution $\left({ }^{*}\right)$ is then a mixture of the guessed times and the 'real' response times:

$$
\begin{aligned}
& P\left\{T_{O^{*}} \leq t\right\}=g P\left\{T_{O} \leq t\right\} \\
& P\left\{T_{A^{*}} \leq t\right\}=g P\left\{T_{O} \leq t\right\}+(1-g) P\left\{T_{A} \leq t\right\} \\
& P\left\{T_{V^{*}} \leq t\right\}=g P\left\{T_{O} \leq t\right\}+(1-g) P\left\{T_{V} \leq t\right\} \\
& P\left\{T_{A V^{*}} \leq t\right\}=g P\left\{T_{O} \leq t\right\}+(1-g) P\left\{T_{A V} \leq t\right\} .
\end{aligned}
$$


$P\left\{T_{0^{*}} \leq t\right\}$ is the observed response time distribution in catch trials. Under the assumption that $\mathrm{g} P\left\{T_{O} \leq t\right\}$ is equal in all stimulus conditions (context independence of the fast guesses), it is easily seen that Inequality (3) is obtained by subtracting $P\left\{T_{0^{*}} \leq\right.$ $t$ \} from the observed distribution functions $P\left\{T_{A^{*}} \leq t\right\}, P\left\{T_{V^{*}} \leq t\right\}$, and $P\left\{T_{A V^{*}} \leq t\right\}$ and dividing by $(1-g)$.

In this note we show that the kill-the-twin procedure can be derived from a more general model of fast guesses in which the guessing state and the responding state are not exclusive (Yellott, 1971, "deadline model"), and we extend the procedure to the case in which redundant stimuli are presented with onset asynchrony. Finally, we show how the kill-the-twin procedure is implemented in Miller's (1986) statistical test of the race model prediction.

\section{Race of fast guess and response}

In the deadline model, a fast guess is considered as a response to a third 'stimulus' (the null stimulus, $O$ ), yielding a second racer in the responses to $A$ and $V$, and a third racer in responses to $A V$ (Yellott, 1971, ch. 9). In a given trial, the observed response time $T_{0^{*}}, T_{A^{*}}, T_{V^{*}}, T_{A V(\tau)^{*}}$ is then the minimum of the response times for the real stimulus $T_{A}, T_{V}, T_{A V(\tau)}$, and the fast guess $T_{O}$.

$T_{0^{*}}={ }_{s t} T_{0}$

$T_{A^{*}}={ }_{s t} \min \left(T_{A}, T_{O}\right)$

$T_{V^{*}}={ }_{s t} \min \left(T_{V}, T_{O}\right)$

$T_{A V(\tau)^{*}}={ }_{s t} \min \left(T_{A}, T_{V}+\tau, T_{O}\right)$.

We again assume context independence, that is, fast guesses are equally probable in $O, A, V$, and $A V(\tau)$. The observed response times for the null stimulus $T_{0^{*}}$ serve as an estimate for $T_{0}$. From (6), the modified race inequality can be derived:

$$
\begin{aligned}
P\left\{T_{A V(\tau)^{*}} \leq t\right\} \leq & P\left\{T_{A^{*}} \leq t\right\}+P\left\{T_{V^{*}}+\tau \leq t\right\} \\
& -P\left\{T_{O^{*}}+\tau \leq t\right\} .
\end{aligned}
$$

For proof, we separately expand both sides of Inequality (7), reiterating the derivation of the race inequality for three stimuli (Diederich, 1992, Eq. 10):

Left:

$$
\begin{aligned}
P\left\{T_{\left.A V(\tau)^{*} \leq t\right\}=}\right. & P\left[\left\{T_{A} \leq t\right\} \cup\left\{T_{V}+\tau \leq t\right\} \cup\left\{T_{O} \leq t\right\}\right] \\
= & P\left\{T_{A} \leq t\right\}+P\left\{T_{V}+\tau \leq t\right\}+P\left\{T_{O} \leq t\right\} \\
& -P\left\{T_{A} \leq t \cap T_{V}+\tau \leq t\right\}-P\left\{T_{A} \leq t \cap T_{O} \leq t\right\} \\
& -P\left\{T_{V}+\tau \leq t \cap T_{O} \leq t\right\} \\
& +P\left\{T_{A} \leq t \cap T_{V}+\tau \leq t \cap T_{O} \leq t\right\} .
\end{aligned}
$$

Right:

$$
\begin{aligned}
& P\left\{T_{A^{*}} \leq t\right\}+P\left\{T_{V^{*}}+\tau \leq t\right\}-P\left\{T_{O^{*}}+\tau \leq t\right\} \\
&= P\left\{T_{A} \leq t\right\}+P\left\{T_{O} \leq t\right\}-P\left\{T_{A} \leq t \cap T_{O} \leq t\right\} \\
&+P\left\{T_{V}+\tau \leq t\right\}+P\left\{T_{O}+\tau \leq t\right\} \\
& \quad P\left\{T_{V}+\tau \leq t \cap T_{O}+\tau \leq t\right\}-P\left\{T_{O}+\tau \leq t\right\} .
\end{aligned}
$$

After eliminating duplicate terms, rearranging and simplifying the notation slightly $\left(T_{A} \rightarrow A\right)$, the inequality reduces to Left:

$$
\begin{gathered}
-P\{A \leq t \cap V+\tau \leq t\}+P\{A \leq t \cap V+\tau \leq t \cap O \leq t\} \\
-P\{V+\tau \leq t \cap O \leq t\} .
\end{gathered}
$$

Right:

$$
-P\{V+\tau \leq t \cap O+\tau \leq t\} .
$$

It is easily seen that the inequality (left $\leq$ right) holds for every $t$ since $-P\{A \leq t \cap V+\tau \leq t\}+P\{A \leq t \cap \bar{V}+\tau \leq t \cap 0 \leq t\} \leq 0$ and $-P\{V+\tau \leq t \cap O \leq t\} \leq-P\{V+\tau \leq t \cap \bar{O}+\tau \leq t\}$.
Thus, the kill-the-twin procedure yields a valid and sharpened upper bound of the race model prediction. The method is easily extended to correct for fast guesses in arbitrary SOA conditions, e.g. A33V. From Inequality (7) it is evident that the impact of fast guesses is vanishing as the SOA is increasing.

We now discuss a few properties of Inequality (7) and the killthe-twin procedure: (a) For choice reactions, Yellott (1971) has demonstrated that the two-state model for fast guesses ("simple fast guess model", SFG) is a special case of the deadline model: In the SFG model, the guessing state and the response state are mutually exclusive, whereas in the deadline model, guess and response are thought to be caused by processes operating in parallel. More formally, assume that the stimulus-controlled response occurs at time $S$. Stimulus-controlled responses occur within the interval $\left[s_{\min }, s_{\max }\right]$. On each trial, the participant sets an internal deadline $D$, at which he or she presses the response key-irrespectively of whether a stimulus has been presented or not. As already shown by Yellott $(1971$, ch. 9$)$ for choice reaction times, the deadline model specializes to the SFG model if $P\{D<$ $\left.s_{\min }\right\}=g, P\left\{s_{\min } \leq D \leq s_{\max }\right\}=0, P\left\{D>s_{\max }\right\}=1-g$, with $g$ again denoting the guessing probability. Of course, Inequality (8) still holds, which is illustrated for synchronous stimuli here: For $t<s_{\min }, P\{A \leq t \cap V \leq t\}=P\{A \leq t \cap V \leq t \cap 0 \leq t\}=0$, for $s_{\min } \leq t \leq s_{\max }, P\{A \leq t \cap V \leq t\}$ increases from 0 to 1 , whereas $P\{A \leq t \cap V \leq t \cap 0 \leq t\}$ increases from 0 to $g$ only, for $t>s_{\max }$, $P\{A \leq t \cap V \leq t \cap O \leq t\}$ increases from $g$ to 1 . The other two terms are identical for $\tau=0: P\{V+\tau \leq t \cap 0 \leq t\}=P\{V+\tau \leq$ $t \cap 0+\tau \leq t\}$.

(b) The derivation of Inequality (7) does not require that the guessing times are stochastically independent from the response times (e.g. Tiefenau, Neubauer, von Specht, \& Heil, 2006). (c) The expression $P\{T \leq t\}$ refers to the "probability that a response occurred within $t$ ms"; the contrary is "no response occurred within $t$ ms", which also covers the case "no response occurred at all". Therefore, this probability is not conditional on the event that the participant actually made a valid response. As a consequence, the distribution function $F(t)=P\{T \leq t\}$ is not necessarily 1 for large $t$, especially for the null stimulus. Rerunning the trials with omitted responses or eliminating omitted responses before measuring $P\{T \leq t\}$ can seriously bias this estimate. (d) Eriksen (1988) suggests subtracting the proportions of fast guesses from each distribution function. As Inequality (7) shows, it is sufficient to subtract $P\left\{T_{O}+\tau \leq t\right\}$ only once from $P\left\{T_{V}+\tau \leq t\right\}$, on the right side of the inequality.

(e) How is the subtraction performed? In Eriksen (1988) the description is somewhat ambiguous ("Thus for each error trial latency, a trial of corresponding latency is subtracted from the CDF of latencies of correct responses.", p. 193), and in fact, eliminating the twins from all three distributions $F_{A}, F_{V}$, and $F_{A V}$ renormalizes the response time distributions by $1 /(1-g)$. In Inequality (7), subtracting $P\left\{T_{O}+\tau \leq t\right\}$ from $P\left\{T_{V}+\tau \leq t\right\}$ might be performed by setting the response time of the twin in $T_{V}$ to infinity, eliminating the response time completely from the distribution would erroneously renormalize the term $P\left\{T_{V}+\tau \leq t\right\}$. (f) Killing a twin as suggested by van der Heijden (see Eriksen, 1988) involves searching for a response to a valid stimulus in a predefined interval around the reaction time of the incorrect fast guess. Two potential problems might arise from such a procedure, especially when small samples are analyzed. First, the procedure loses its effectiveness when a valid twin with similar response time is missing. Second, Inequality (7) might accidentally be violated if the decrease of $F_{A V}(t)$ due to the twin killing is smaller than the decrease of $F_{A}(t)$ and $F_{V}(t)$. For a given response time $t_{0}$ to a null stimulus, this might happen if the twin in $T_{A V}$ is greater than $t_{0}$, whereas the twins in $T_{A}$ and $T_{V}$ are both smaller than $t_{0}$. As already pointed out in (d), the two minuends $P\left\{T_{0^{*}} \leq t\right\}$ on the left and on the 
right side of Inequality (7) cancel out. It is, therefore, not necessary to correct the distribution functions $F_{A V}(t)$ and $F_{A}(t)$; this should ameliorate the problem. For the remaining term, $P\left\{T_{V^{*}}+\tau \leq t\right\}$, two correction strategies can be distinguished. If the race model prediction is to be rejected in a given experiment, an accidental violation of the race inequality is avoided using a conservative correction procedure. For a given fast guess $t_{0}$, only a response time greater than or equal to $t_{0}$ is 'killed'. A progressive correction would kill only a response time smaller than or equal to $t_{0} \cdot{ }^{1}$

A non-trivial assumption of the kill-the-twin correction is that the latent distribution of guessing times $P\left\{T_{0} \leq t\right\}$ is equal in all stimulus conditions $(O, A, V, A V)$. In a simple reaction time experiment with a randomized sequence of stimuli, the probability for a fast guess should be context independent. Is there a possibility to test this assumption? Consider, for example, an experiment similar to Posner (1980) in which an arrow indicates the possible location of an upcoming target stimulus $(O, A, V, A V)$. It is well plausible that despite the catch trials and a variable interval between the cue and the target, the participant might be encouraged by the paradigm to anticipate the onset of the target stimulus. A serious violation of context independence might be detected by defining a minimal response time (e.g. $100 \mathrm{~ms}$ after target onset) and by comparing the proportions of responses in the interval between cue onset and target onset $+100 \mathrm{~ms}$ in the different conditions. To avoid that the race model is erroneously rejected, the number of fast guesses observed in the bimodal condition should not substantially exceed the number of fast guesses observed in the unimodal conditions.

\section{Statistical test}

Miller (1986) has suggested a procedure for a statistical test of the race inequality; with one exception (Schwarz, 2006) this procedure has been widely disregarded despite its appealing logic. We reiterate it here and we adapt it to include the correction for fast guesses. In Miller's (1986) test, the statistic of interest is the 'violation area', that is, the area between $F_{A V}(t)$ and $F_{A}(t)+$ $F_{V}(t)$, where $F_{A V}(t)$ exceeds $F_{A}(t)+F_{V}(t)$. More formally, define a function $g(t)$ and an area $V$ as:

$g(t)=F_{A V}(t)-\min \left[1, F_{A}(t)+F_{V}(t)\right]$

$V=\int_{0}^{\infty} \max [0, g(t)] \mathrm{d} t$.

Colonius and Diederich (2006) have shown that $\int_{0}^{\infty} g(t) \mathrm{d} t$ is equal to the difference between the observed mean response time for $A V$ and the mean response time obtained by a race model with maximally negative channel correlation. The violation area $V_{\text {obs }}$ as defined by (9) can be determined numerically using the empirical cumulative distribution functions. To determine whether $V_{\text {obs }}>$ 0 is due to sampling error, the experiment is simulated 10,000 times. In each simulation, response times for $A$ and $V$ are sampled with replacement from the observed response time distributions ('bootstrap'). For responses to $A V$, 'antithetic' pairs of auditory and visual response times are drawn, and the smaller of the two values is chosen (race model). Antithetic means that the auditory response time at percentile $P$ is accompanied by the visual response time at percentile $100-P$ (Miller, 1986, p. 337). Such a negative channel correlation minimizes the intersection $\left\{T_{A} \leq t\right\}$

\footnotetext{
${ }^{1}$ The above description of the kill-the-twin procedure implicitly assumes that the number of trials is equal in all stimulus conditions. If the number of trials differs between conditions, e.g. $N_{V}: N_{O}=3: 1$, three twins have to be killed for each fast guess. More generally, conservative correction: floor $\left(N_{V} / N_{O}\right)$, progressive: ceiling $\left(N_{V} / N_{O}\right)$.
}

$\cap\left\{T_{V} \leq t\right\}$, hence the bootstrapped response time distribution for $A V$ is closer to $F_{A}(t)+F_{V}(t)$ (Miller, 1982, p. 253). In each simulation $\mathrm{i}$, a violation area $V_{i}$ is determined according to (9). Following the logic of a statistical null hypothesis test, the race model is rejected with $P=.05$ if $V_{\text {obs }}$ is greater than $95 \%$ of the simulated $V_{i}$.

Miller's (1986) test is easily extended to account for fast guesses. To avoid the kill-the-twin correction accidentally resulting in a violation of Inequality (3), the observed violation area $V_{\text {obs }}$ is determined by using the conservative kill-the-twin correction (see above, $f$ ). In contrast, the simulation of the experiment is performed by using the response times corrected by the progressive kill-the-twin procedure; this ensures that the size of the simulated violation areas $V_{i}$ is sufficient. This procedure should keep the type I error below the predefined limit, while the refined upper bound of Inequality (7) should, in any case, improve the power of the testing procedure.

\section{Application}

In most experiments, anticipatory responses are avoided by instruction and by design (e.g. by variable inter-stimulus-intervals). Nevertheless, there are situations in which the participant might be encouraged to anticipate the onset of the stimulus, for example, in the Posner-like cuing task described above. In such experiments, it is very difficult to demonstrate a violation of the race model prediction without the kill-the-twin correction. As Miller and Lopes (1991, Fig. 2, upper panel), have shown using simulations, even a low number of fast guesses $(g=0.02)$ can seriously bias the outcome of the test of Inequality (3). The effect of the killthe-twin correction is also seen in Hughes, Reuter-Lorenz, Nozawa, and Fendrich (1994). From figure 5 (Hughes et al., 1994, p. 137), it is evident that the violation of the race inequality is completely masked by the distribution of fast guesses.

Among the 305 articles which refer to Miller's article from 1982 (Aug. 2008, ISI web of knowledge), only a small number refer to the kill-the-twin procedure (KTT) suggested by Eriksen (1988). Closer inspection of these articles reveals that only 10 of them actually used it. Two potential reasons come to mind: First, the KTT is derived from specific assumptions, namely, the simple fast guess model (Ollman, 1966) with two exclusive states, a guess state and a response state. In the derivation of Inequality (7), we show that the KTT can be derived from a more general model in which the guess and response processes operate in parallel, the assumptions can, therefore, be relaxed to some extent. Second, there is little need for a correction procedure if only a few anticipatory responses are observed or if the violation of Inequality (3) is large, for example, with auditory-visual redundant signals. In contrast, in unimodal tasks (for example, redundant targets within the visual modality, Feintuch \& Cohen, 2002), coactivation effects are generally small or absent. In such a situation, the kill-thetwin procedure might help distinguishing parallel from coactive processing (e.g. Krummenacher, Müller, \& Heller, 2001).

\section{Acknowledgments}

We are grateful to Hans Colonius and to an anonymous reviewer for helpful comments on an earlier version of the manuscript.

\section{References}

Colonius, H., \& Diederich, A. (2006). Race model inequality: Interpreting a geometric measure of the amount of violation. Psychological Review, 113, 148-154.

Diederich, A. (1992). Probability inequalities for testing separate activation models of divided attention. Perception \& Psychophysics, 52, 714-716.

Eriksen, C. W. (1988). A source of error in attempts to distinguish coactivation from separate activation in the perception of redundant targets. Perception $\mathcal{E}$ Psychophysics, 44, 191-193.

Feintuch, U., \& Cohen, A. (2002). Visual attention and coactivation of response decisions for features from different dimensions. Psychological Science, 13 361-369.

Hughes, H. C., Reuter-Lorenz, P. A., Nozawa, G., \& Fendrich, R. (1994). Visual-auditory interactions in sensorimotor processing. Saccades versus manual responses. 
Journal of Experimental Psychology: Human Perception and Performance, 20, 131-153.

Krummenacher, J., Müller, H., \& Heller, D. (2001). Visual search for dimensionally redundant pop-out targets: Evidence for parallel-coactive processing of dimensions. Perception \& Psychophysics, 63, 901-917.

Luce, R. D. (1986). Response times. Their role in inferring mental organization. New York: Oxford University Press.

Maris, G., \& Maris, E. (2003). Testing the race model inequality: A nonparametric approach. Journal of Mathematical Psychology, 47, 507-514.

Miller, J. O. (1982). Divided attention: Evidence for coactivation with redundant signals. Cognitive Psychology, 14, 247-279.

Miller, J. O. (1986). Timecourse of coactivation in bimodal divided attention. Perception \& Psychophysics, 40, 331-343.
Miller, J., \& Lopes, A. (1991). Bias produced by fast guessing in distribution-based tests of race models. Perception \& Psychophysics, 50, 584-590.

Ollman, R. (1966). Fast guesses in choice reaction time. Psychonomic Science, 6, $155-156$.

Posner, M. (1980). Orienting of attention. Quartely Journal of Experimental Psychology, 32, 3-25.

Schwarz, W. (2006). On the relation between the redundant signals effect and temporal order judgments: Parametric data and a new model. Journal of Experimental Psychology: Human Perception and Performance, 32, 558-573.

Tiefenau, A., Neubauer, H., von Specht, H., \& Heil, P. (2006). Correcting for false alarms in a simple reaction time task. Brain Research, 1122, 99-115.

Yellott, J. I., Jr. (1971). Correction for fast guessing and the speed-accuracy tradeoff in choice reaction time. Journal of Mathematical Psychology, 8, 159-199. 